\title{
Overexpression miR-486-3p Promoted by Allicin Enhances Temozolomide Sensitivity in Glioblastoma Via Targeting MGMT
}

\author{
Henggang $\mathrm{Wu}^{2} \cdot \mathrm{Xu} \mathrm{Li}^{3} \cdot$ Tiehui Zhang ${ }^{3} \cdot$ Guojun Zhang ${ }^{1}$. Jingnan Chen ${ }^{1} \cdot \mathrm{Li} \mathrm{Chen}^{1} \cdot \mathrm{Min} \mathrm{He}^{1} \cdot$ Bilie Hao ${ }^{1}$. \\ Cheng Wang ${ }^{1}$ (1)
}

Received: 26 September 2019 / Accepted: 3 February 2020 / Published online: 21 February 2020

(c) The Author(s) 2020

\begin{abstract}
Glioblastoma is the most common primary tumor of the central nervous system that develops chemotherapy resistance. Previous studies showed that Allicin could inhibit multiple cancer cells including glioblastoma, but the function of Allicin in glioblastoma is still unclear. Our work aimed to investigate the underlying molecular mechanism. The results showed that miR-486-3p levels were greatly increased in glioblastoma during Allicin treatment. Overexpression of miR-486-3p increased chemosensitivity to temozolomide (TMZ) in vitro and in vivo. O6-methylguanine-DNA methyltransferase (MGMT) was identified as a direct target of miR-486-3p, and miR-486-3p overexpression prevented the protein translation of MGMT. Moreover, overexpression of MGMT restored miR-486-3p-induced chemosensitivity to TMZ. Taken together, our studies revealed that Allicin could upregulate miR-486-3p and enhance TMZ sensitivity in glioblastoma. The results suggested that in the future, Allicin can be used as an adjuvant therapy with TMZ to improve the prognosis of patients, and miR-486-3p may be a potential target for glioblastoma treatment to improve the curative effects.
\end{abstract}

Keywords Allicin · O6-methylguanine-DNA methyltransferase (MGMT) $\cdot$ Temozolomide (TMZ) $\cdot$ Glioblastoma $\cdot$ miR486-3p

\section{Introduction}

Glioblastoma (GBM) are the most common central nervous system tumors in the clinic, accounting for $46.6 \%$ of all primary central nervous system tumors (Ostrom et al. 2016; Bush et al. 2017). At present, the treatment to glioblastoma

Henggang $\mathrm{Wu}$ and $\mathrm{Xu} \mathrm{Li}$ have contributed equally to this work.

Electronic supplementary material The online version of this article (https://doi.org/10.1007/s12017-020-08592-5) contains supplementary material, which is available to authorized users.

Cheng Wang

xh_wangcheng@163.com

1 Department of Neurosurgery, The Second Affiliated Hospital of Zhejiang Chinese Medical University, Hangzhou 310011, Zhejiang, China

2 Department of Neurosurgery, Wenrong Hospital of Hengdian, Jinhua 322118, Zhejiang, China

3 Department of Neurosurgery, The First Affiliated Hospital of Zhejiang Chinese Medical University, Hangzhou 310002, Zhejiang, China is mainly based on surgical resection, post-operative radiotherapy, and chemotherapy as adjuvant therapy. Adjuvant therapy can significantly prolong the median survival of glioblastoma patients and improve their quality of life.

Temozolomide (TMZ) has been the standard chemotherapy for newly diagnosed GBM for many years. TMZ has certain advantages over many existing alkylating agents because of its small molecular weight. Many studies have shown that the levels of drug in the brain and cerebrospinal fluid are approximately $30 \%$ to $40 \%$ of the plasma concentration. Although TMZ can prolong the survival time by a small margin, a considerable number of glioblastoma patients are insensitive to TMZ treatment or gradually develop secondary resistance. Therefore, glioblastoma resistance to TMZ is considered to be the underlying cause of chemotherapy failure and glioblastoma recurrence (Kang et al. 2004). The underlying mechanisms require investigation and solutions to the problem of drug resistance are being sought.

MicroRNAs (miRNAs) are involved in a variety of human diseases, which can regulate gene expression posttranscriptionally by binding to mRNA 3'-untranslated region (Iorio and Croce 2009; Omura et al. 2008). Previous study 
demonstrated that miR-486 is often downregulated in malignancies and considered to be a tumor-suppressor miRNA (Chou et al. 2019; Yang et al. 2019). In addition, miR-486 could be an effective biomarker in cancer diagnosis and prognosis (Jiang et al. 2018). According to Genetics Network Analysis, Plaisier et al. found that high expression of miR-486-3p may enhance the sensitivity of neuronal tumors to chemotherapeutic drugs (Plaisier et al. 2016). However, this hypothesis has not been proved, and the effects of miR486-3p on chemotherapeutic sensitivity of glioblastoma to TMZ and the underlying mechanisms are unknown.

Allicin is an ubiquitously found ingredient in garlic and widely used as food supplements all over the world(Lawson and Wang 2005). Allicin is reported to induce cell death and inhibit proliferation in cancer cells (Borlinghaus et al. 2014). These studies also suggested that Allicin induced p53-mediated autophagy in human liver cancer cells (Chu et al. 2012). Although studies have found that Allicin can inhibit glioblastoma proliferation (Pan et al. 2018), whether Allicin can enhance the sensitivity of glioblastoma to TMZ is unknown. Therefore, this study selected glioblastoma cell lines U251-TR, which is resistant to TMZ, as a research object to explore whether Allicin can reverse the TMZ resistance and was also performed to investigate the mechanism.

\section{Materials and Methods}

\section{Cell Lines and Cell Culture}

The glioblastoma cell lines U251-MG and A172 were obtained from the Cell Bank of the Chinese Academy of Sciences (Shanghai, China). The cell line was cultured in Dulbecco's modified Eagle's medium (DMEM, Gibco, Carlsbad, CA, USA) containing $2 \mathrm{mM}$ glutamine, $10 \%$ fetal calf serum (FBS), $100 \mathrm{U} / \mathrm{ml}$ penicillin and $100 \mu \mathrm{g} / \mathrm{ml}$ streptomycin (Sigma, St. Louis, MO, USA). Cells were maintained at $37^{\circ} \mathrm{C}$ in a $5 \% \mathrm{CO}_{2}$ incubator.

\section{Isolation and Culture of Primary Glioblastoma Cells}

Fresh brain tumor samples were collected from adult patients at the time of surgical excision who had provided informed written consent, and the tumors were removed surgically according to guidelines approved by the Institutional Review Board of The Second Affiliated Hospital of Zhejiang Chinese Medical University. GBM cells were isolated as described in previous study (Shankar et al. 2014; Rahman et al. 2015). Tumor specimens were washed thoroughly with cold PBS and cut into $1 \mathrm{~mm}^{3}$ pieces, then digested with trypsin $(0.5 \%, \mathrm{~V} / \mathrm{M})$ for $20-40 \mathrm{~min}$ at $37{ }^{\circ} \mathrm{C}$ in a shaking water bath. Primary glioblastoma cells were gently resuspended in DMEM/F12 medium ( $2 \mathrm{mmol} / 1 \mathrm{~L}$-glutamine $)$ supplemented with $0.05 \%$ BSA, $20 \mathrm{ng} / \mathrm{ml} \mathrm{bFGF}, 10 \mathrm{ng} / \mathrm{ml}$ EGF, $50 \mathrm{U} \mathrm{ml}$ of penicillin, and $50 \mu \mathrm{g} \mathrm{ml}$ of streptomycin. The culture medium was refreshed every 2-3 days.

\section{Generation of Temozolomide-Resistant Glioblastoma Cell Lines}

The parental U251-MG cells were exposed to $400 \mu \mathrm{M}$ TMZ for 3 weeks to generate TMZ-resistant colonies (Xu et al. 2017). Initially, we cultured the cell line in 6-well plates and allowed them to adhere during overnight incubation at $37^{\circ} \mathrm{C}$. TMZ treatment was repeated every $24 \mathrm{~h}$ for 5 consecutive days, and the cells were then exposed to fresh TMZ every 3 days for a total of 3 weeks. The majority of the cells died, but a small population survived and propagated. The surviving colonies were selected and established as TMZresistant U251 (U251-TR) cell lines.

\section{RNA Extraction, Reverse Transcription, and Quantitative-PCR (q-PCR)}

Total RNAs were isolated using TRIzol (Ambion) based on the manufacturer's protocol. For mRNA analysis, the cDNA was synthesized using random hexamer primers and SuperScript III reverse transcriptase (Invitrogen, Carlsbad, CA). For miRNA analysis, the cDNA was synthesized using specific stem-loop RT primers and TaqMan MicroRNA Reverse Transcription Kit (Applied Biosystems). q-PCR analysis was used to detect the MGMT using Maestro Green Eva Green q-PCR Master Mix (Maestrogen) and the expression level of miR-486-3p using QuantiTect SYBR Green PCR System (QIAGEN), according to the manufacturer's instructions on the ABI Step OnePlus Real-time PCR system (Applied Biosystems). GAPDH was used as the internal control (Table 1). All reactions were run in triplicate and relative expression levels were calculated as $2^{-\Delta \Delta C_{\mathrm{T}}}$ after normalization with internal control.

\section{Plasmid Construction and Luciferase Reporter Assay}

The target genes of miR-486-3p were selected based on the TargetScan (https://www.targetscan.org/, Release 7.2: March

Table 1 Sequences of primers used for experiments in this study

\begin{tabular}{ll}
\hline Primer name & Sequence $\left(5^{\prime}-3^{\prime}\right)$ \\
\hline GAPDH & F: GAAGGTGAAGGTCGGAGT \\
& R: GAAGATGGTGATGGGATTTC \\
MGMT & F: ACCGTTTGCGACTTGGTACTT \\
& R: GGAGCTTTATTTCGTGCAGACC \\
miR-486-3p & F: GCGGGGCAGCTCAGTA \\
& R: CGGGGCAGCTCAGTACAGGAT \\
\hline
\end{tabular}


2018). MGMT was selected as the possible target gene of miR-486-3p. The entire 3'-UTR of MGMT fragment, containing target sequences of miR-486-3p, were PCR amplified and cloned into the pmirGLO firefly luciferase-expressing vector (Promega, WI, USA) according to the manufacturer's instructions. The miR-486-3p binding site mutation vectors were also constructed by using Site-Directed Mutagenesis Kit (Stratagene, La Jolla, CA), and all the constructs were verified by DNA sequencing. For luciferase reporter assay, luciferase reporter vectors together with miRNA-mimics (PM-486-3p) and negative control (NC) were transfected into U251-TR cells using Lipofectamine RNAi MAX Transfection Reagent (Thermo Fisher). After $48 \mathrm{~h}$, luciferase activities were detected using the Dual Luciferase Reporter Assay System (Promega) on the Orion L luminometer (Berthold), according to the manufacturer's protocol. Renilla luciferase served as the control reporter for normalization.

\section{Cytotoxicity Assay}

Cell viability was measured using a cell counting kit-8 colorimetric assay (CCK-8; Dojindo Laboratories, Kumamoto, Japan) according to the manufacturer's instructions. The transfected or wild-type cells were seeded into 96-well plates ( 3000 cells/well) and incubated at $37^{\circ} \mathrm{C}$. After $24 \mathrm{~h}$, $10 \mu \mathrm{L}$ of CCK- 8 reagent was added per well and the cells were maintained for $2 \mathrm{~h}$. The optical density values were detected at $450 \mathrm{~nm}$ using a Multiskan Sky Microplate Spectrophotometer (Thermo Fisher Scientific). IC $_{50}$ values were determined using GraphPad Prism software version 5.0 (GraphPad Software, San Diego, CA, USA).

\section{Cell Apoptosis Assay}

For cell apoptosis assay, $2 \times 10^{5}$ cells were seeded in 6-well plates and transfected with miR-486-3p mimics (PM486-3p) and negative control (NC). The cell apoptosis was detected according to the instructions of Annexin V-fluorescein isothiocyanate (FITC) kit (Sigma, St. Louis, MO, USA). Double staining with FITC-conjugated annexin V and propidium iodide (PI) was performed as follows. Forty-eight hours post transfection, the cells, including floating cells, were harvested, washed twice with $4{ }^{\circ} \mathrm{C}$ PBS, and resuspended in binding buffer (10 mM HEPES/NaOH, $140 \mathrm{mM}$ $\mathrm{NaCl}, 2 \mathrm{mM} \mathrm{KCl}$ ). Annexin $\mathrm{V}$ was added for $15 \mathrm{~min}$ in the dark. Cells were then washed, centrifuged, and resuspended in binding buffer. Before flow cytometric analysis, PI was added to each sample. Annexin V+/PI-cells were early apoptotic cells. Finally, the cell apoptosis detection was performed by a FACS Canto II flow cytometer (Becton Dickinson, Franklin Lakes, NJ, USA).

\section{Western Blot Analysis}

Total protein was extracted from cells in one well of a 6 -well plate $\left(\sim 1 \times 10^{6}\right.$ cells $)$ following $24 \mathrm{~h}$ treatment with $100 \mu \mathrm{M} \mathrm{TMZ}$ as aforementioned and the concentrations were measured using a spectrophotometer (Bio-Rad Laboratories, Inc., Hercules, CA, USA) with a bicinchoninic acid protein assay kit (Beyotime Institute of Biotechnology, Shanghai, China). Protein at a concentration of $>5 \mu \mathrm{g} / \mu \mathrm{l}(50 \mu \mathrm{g}$ in total) was separated by $10 \%$ or $12 \%$ SDS-PAGE and was transferred onto polyvinylidene difluoride membranes (EMD Millipore, Billerica, MA, USA), followed by blocking with skimmed milk dissolved in TBS and $0.05 \%$ Tween-20 (TBST) for $1 \mathrm{~h}$ at room temperature. The membranes were incubated with primary antibodies including MGMT (sc-271154, Santa Cruz, CA, USA), caspase-3 (9661S, Cell Signaling Technology, USA), $\gamma$-H2AX (2577S, Cell Signaling Technology, USA), and H2AX (2572, Cell Signaling Technology, USA) at $4{ }^{\circ} \mathrm{C}$ overnight, washed three times with TBST and were incubated in horseradish peroxidase-conjugated secondary antibodies at 1:5000 dilution (sc-2004; Santa Cruz Biotech, Santa Cruz, CA, USA) for $1 \mathrm{~h}$ at room temperature after washing with TBST three times. Following washing, the protein bands were detected with ECL substrates or DAB Detection System (OriGene Technologies, Inc., Beijing, China). $\beta$-actin (sc-47778, Santa Cruz, USA) was used as a loading control, and all experiments were repeated three times. Quantitative analysis was performed using the Quantity One Software (version 4.6.2; Bio-Rad Laboratories, Inc.).

\section{Nude Mouse Tumorigenicity Assay}

Tumor-bearing nude mice (weight: 18-20 g; Vital River, Beijing, China) were divided into three groups (six mice per group). U251-TR cells $\left(2 \times 10^{6}\right)$ stably expressing miR486-3p, miR-486-3p-inhibitor, and miR-NC were collected and injected subcutaneously into NOD/SCID mice. All mice were housed under standard specific pathogen-free

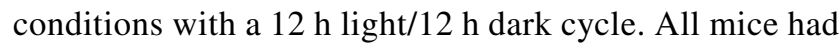
free access to food and water. After 2 weeks of tumor growth, all mice were given TMZ + Allicin $(60 \mathrm{mg} / \mathrm{kg}$ $\mathrm{TMZ}+50 \mathrm{mg} / \mathrm{kg}$ Allicin, oral gavage). The mice were treated daily for 3 weeks. Mice were monitored visually and sacrificed when neurological signs appeared. Kaplan-Meier survival curves were calculated using GraphPad Prism 7.0 software. This animal experiment was approved by the Institutional Review Board of The 
Second Affiliated Hospital of Zhejiang Chinese Medical University.

\section{Statistical Analysis}

Quantitative data are presented as means \pm SEM. The statistical significance levels were set at $* P<0.05$ and $* * P<0.01$. One-way ANOVA and Student's $t$ tests were used to perform comparisons. Biostatistical analysis was performed using Office SPSS software (SPSS version 22.0) and GraphPad software (GraphPad Prism 5). All statistical tests were two-sided.

\section{Results}

\section{Allicin Enhances the Sensitivity of U251-TR Cells to TMZ and Promotes Apoptosis}

We firstly established TMZ-resistant GBM cell lines with increased concentrations of TMZ for different time periods (TMZ-resistant U251 cell lines: U251-TR). U251-TR and Parental U251 cells were treated with different concentrations $(0,10,20,50,100$, and $200 \mu \mathrm{g} / \mathrm{ml})$ of TMZ for 24,48 , or $72 \mathrm{~h}$. Results from CCK-8 assays showed that U251-TR cell viability was not affected when TMZ concentrations were $<50 \mu \mathrm{g} / \mathrm{ml}$ within $24 \mathrm{~h}$. Whereas when the TMZ was increased to $200 \mu \mathrm{g} / \mathrm{ml}$ or extended to $72 \mathrm{~h}$, there was no significant decrease in cell viability compared to the $50 \mu \mathrm{g} /$ $\mathrm{ml}$ (Fig. 1a, b). However, parental U251 cell viability significantly decreased in a dose-and time-dependent manner. These results indicated that TMZ-resistant GBM cell line (U251-TR) was successfully established. In addition, U251-TR and Parental U251 cells were treated with different concentrations $(0,10,20,30,40$, and $50 \mu \mathrm{g} / \mathrm{ml})$ of Allicin for 24, 48, or $72 \mathrm{~h}$. Results showed that Allicin did not affect cell viability at concentrations less than $20 \mu \mathrm{g} / \mathrm{ml}$ within $24 \mathrm{~h}$, whereas higher concentrations $(>30 \mu \mathrm{g} / \mathrm{ml})$ of Allicin inhibited both U251-TR and parental U251 cell growth at 48

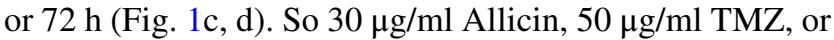
combination $(30 \mu \mathrm{g} / \mathrm{ml}$ Allicin $+50 \mu \mathrm{g} / \mathrm{ml} \mathrm{TMZ})$ were used in the following study.

To determine whether Allicin can enhance the sensitivity of glioblastoma to TMZ. U251-TR and parental U251 cells were treatment with $50 \mu \mathrm{g} / \mathrm{ml} \mathrm{TMZ}$ and/or $30 \mu \mathrm{g} / \mathrm{ml}$ Allicin for 24,48 , or $72 \mathrm{~h}$, The results showed that $30 \mu \mathrm{g} / \mathrm{ml}$ Allicin $+50 \mu \mathrm{g} / \mathrm{ml} \mathrm{TMZ} \mathrm{can} \mathrm{significantly} \mathrm{decrease} \mathrm{parental}$ U251 and U251-TR cells viability in time-dependent manner (Fig. 1e, f). Furthermore, primary glioblastoma cells were also used to confirm this effect, the results showed that $30 \mu \mathrm{g} / \mathrm{ml}$ Allicin $+50 \mu \mathrm{g} / \mathrm{ml} \mathrm{TMZ}$ also can significantly decrease primary glioblastoma cells viability (Supplementary Fig. 1).
In addition, Annexin V-FITC/PI staining assay was used to measure the apoptosis. The results in Fig. 2a show that compared with control, the percentage of apoptotic cells in combination $(30 \mu \mathrm{g} / \mathrm{ml}$ Allicin $+50 \mu \mathrm{g} / \mathrm{ml} \mathrm{TMZ})$ group significantly increased from $5.78 \%$ of the control, $30.19 \%$ of $30 \mu \mathrm{g} / \mathrm{ml}$ Allicin, or $34.35 \%$ of $50 \mu \mathrm{g} / \mathrm{ml} \mathrm{TMZ}$ to $51.49 \%$ (Fig. 2b). Taken together, these results indicated that Allicin can enhance the sensitivity of glioblastoma to TMZ.

\section{Allicin Enhances the Sensitivity of U251-TR Cells to TMZ by Promoting the Expression of miR-486-3p}

Previous study showed that high expression of miR-486-3p may enhance the sensitivity of neuronal tumors to chemotherapeutic drugs. So we explored the levels of miR-486-3p in Allicin group and control group using qRT-PCR. The results showed that miR-486-3p levels were higher in Allicin group than control group (Fig. 2c). In order to verify whether Allicin improves the sensitivity of U251-TR to TMZ by regulating miR-486-3p, U251-TR cells were transfected with lentivirus vectors carrying miR-486-3p or inhibitor. An empty virus was used as positive transfection control (NC) group, and all groups were treated by $30 \mu \mathrm{g} /$ $\mathrm{ml}$ Allicin $+50 \mu \mathrm{g} / \mathrm{ml} \mathrm{TMZ}$. As the results demonstrated, miR-486-3p group had lowest cell viability, but miR-486-3pinhibitor group had highest cell viability (Fig. 2d). This indicated that Allicin could promote miR-486-3p overexpression, then enhances TMZ sensitivity in glioblastoma.

\section{MiR-486-3p Directly Targets MGMT}

To better understand the underlying function and mechanism of miR-486-3p in glioblastoma progression, several potential target genes of miR-486-3p were detected using the bioinformatic algorithm TargetScan (https://www.targetscan .org/). Among the candidates, we detected a predicted binding site for miR-486-3p in the 3'-UTR of MGMT (Fig. 3a). To verify whether MGMT is a novel direct target of miR486-3p in glioblastoma, a luciferase assay was performed in pGL3-control luciferase reporter plasmids containing the putative wild-type (WT) and mutant (Mut) MGMT 3'-UTR binding site. U251-TR cells were treated with reporter vector (MGMT-WT or MGMT-Mut) and miR-NC (negative control) or miR-486-3p. Cells co-transfected with miR-486-3p and MGMT-WT reporter showed a noteworthy reduction of luciferase activities (Fig. 3b).

To further verify whether miR-486-3p regulates MGMT expression through mRNA degradation or translational repression, we established U251-TR cells stably expressing miR-486-3p or miR-NC. And the protein levels of MGMT were downregulated in cells stably expressing miR-486-3p (Fig. 3c). However, miR-486-3p overexpression did not affect the mRNA levels of MGMT (Fig. 3d). These results 


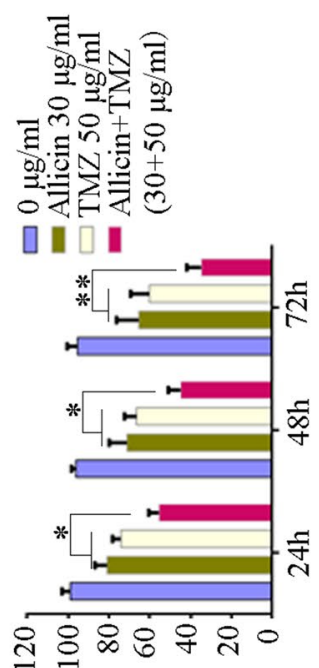

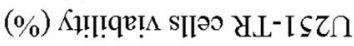

되
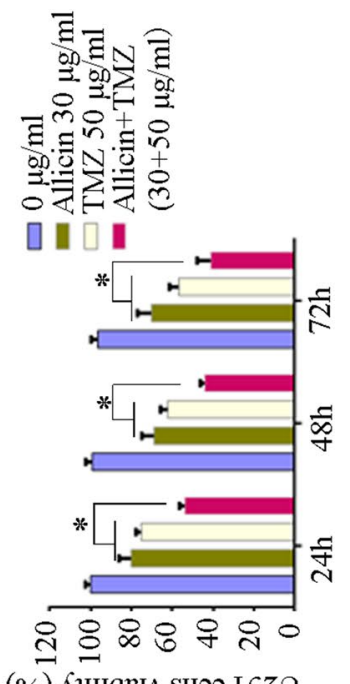

(\%) К‼!qu!

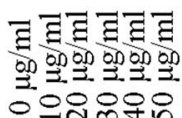

प11010

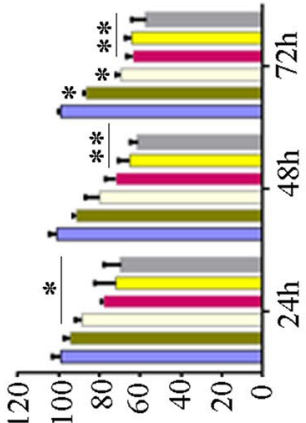

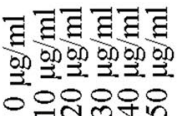

101010

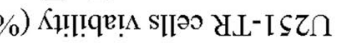

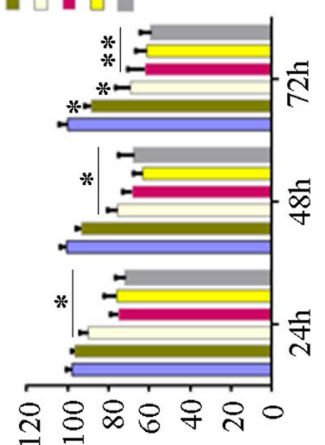

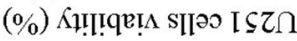

ปั่

응

ญ 屯

च

寻 $\stackrel{\circ}{*}$

造 2

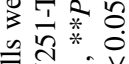

ชุ 2 ह

ธี

กิ

要灵安

ขึㅇํㅇ

चै ठै.

卷选

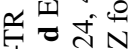

实它既

ऽิ

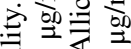

은 웅

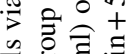

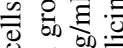

훙

촗요

సै चี

च्च के के

ํํㄴ

ت유요

ㄴํㄹ

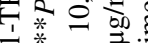

ล่

节

늘

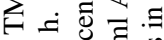

प듕

论

迎㐫氙怘

웝

ธิ

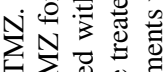

动

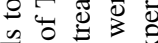

ฮิ

व

王至

공

○司焉

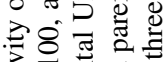

을 믈

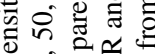

क 尺ें

은

过戞

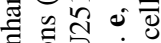

ए)

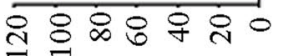

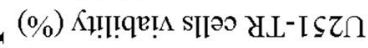

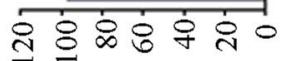

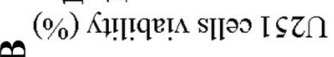

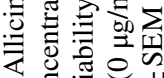

- ठำ

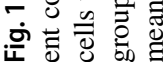



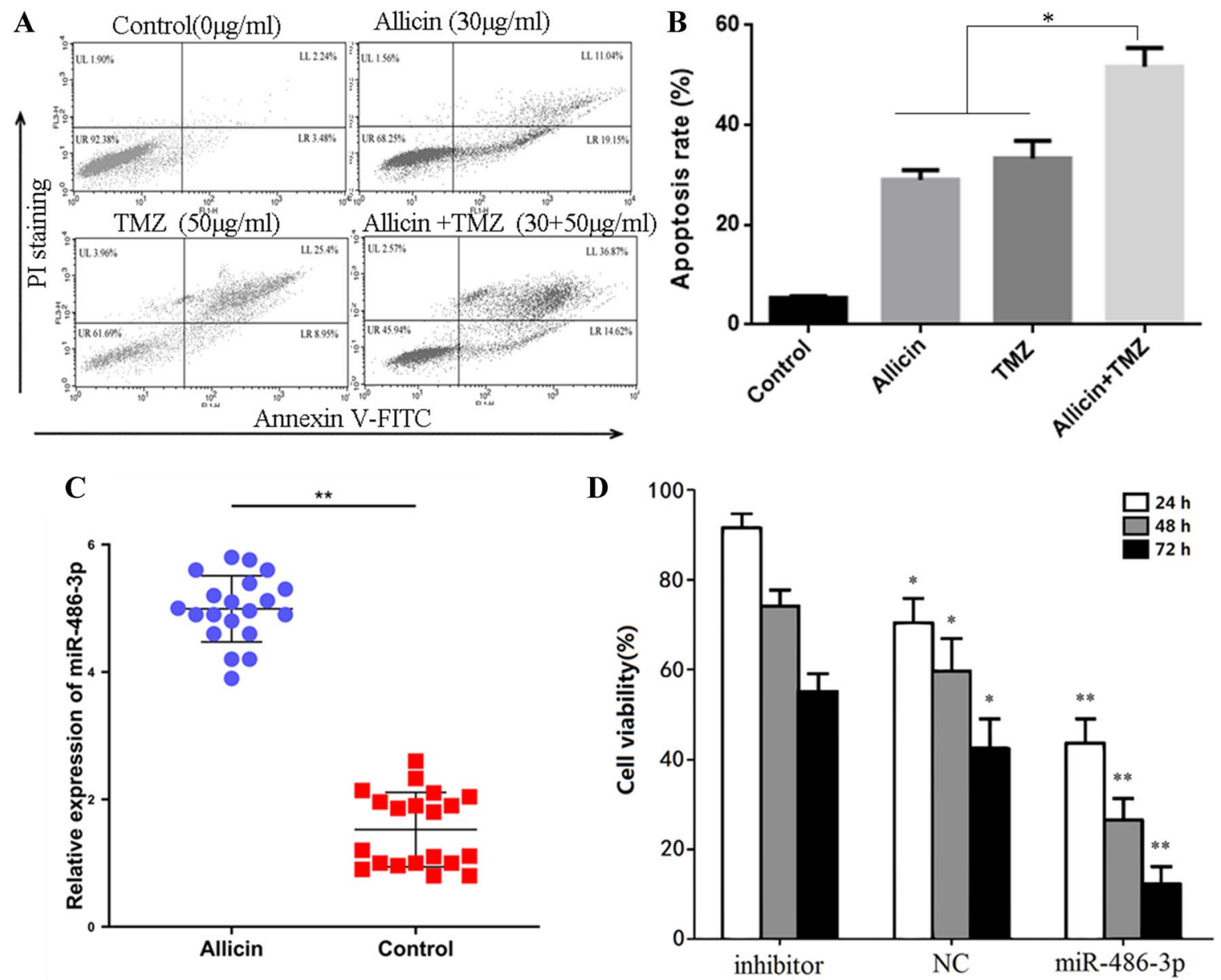

Fig. 2 Allicin enhances the sensitivity of U251-TR cells to TMZ and promotes apoptosis and the expression of miR-486-3p. a Flow cytometry analysis showed that $\mathrm{TMZ}+$ Allicin group could significantly enhance apoptosis in cells after $48 \mathrm{~h}$. b PCR analysis of miR-486-3p expression levels in Allicin group and control group. c Cell viability

analyzed after treatment with Allicin + TMZ in control, miR-486-3p group, miR-486-3p-inhibitor group. Data are mean \pm SEM from three independent experiments with triplicates in each experiment. $* P<0.05, * * P<0.01$

indicated that miR-486-3p directly targeted MGMT by binding to the 3'-UTR of MGMT and inhibited the translation of the MGMT mRNA into the MGMT protein in glioblastoma cells. These findings suggest a possible relationship between miR-486-3p expression and TMZ resistance.

\section{MiR-486-3p Overexpression Increases Chemosensitivity to Temozolomide}

A172 cells are TMZ-sensitive cells lacking MGMT expression. We treated A172 cells and U251-TR cells stably expressing miR-486-3p or miR-NC with different concentrations of TMZ. As shown in Fig. 4a, CCK8 assay detected a significant decrease in cell viability following TMZ exposure in inverse correlation to TMZ concentration. Overexpressing miR-486-3p only increased chemosensitivity to TMZ in U1251-TR cells, but not in A172

cells. Next, we measured the viability of glioblastoma cells treated with TMZ $(200 \mu \mathrm{g} / \mathrm{ml})$ using CCK8 assay at different time points. The results showed that miR-486-3p overexpression significantly inhibited cell survival of U251-TR cells in the presence of TMZ (Fig. 4b). However, overexpression of miR-486-3p did not affect cell survival of A172 cells, indicating that miR-486-3p sensitized glioblastoma cells to TMZ might through MGMT. In addition, after TMZ treatment, glioblastoma cells expressing miR-486-3p had a significant decrease in the expression of MGMT and increase in the levels of phosphorylated histone $\mathrm{H} 2 \mathrm{AX}(\gamma-\mathrm{H} 2 \mathrm{AX}$, Markers of DNA double strand breakage) and cleaved caspase-3 compared with vector control cells. However, ectopic expression of MGMT significantly reversed the enhancement effect of miR-486-3p on $\gamma-\mathrm{H} 2 \mathrm{AX}$ and cleaved caspase- 3 in the presence of TMZ (Fig. 4c). 
Fig. 3 miR-486-3p directly targets MGMT. a The sequence of miR-486-3p binding sites within MGMT. The reporter constructs of the MGMT 3'-UTR sequences. b Luciferase reporter assay was performed to detect the relative luciferase activities of WT and Mut MGMT reporters. $\mathbf{c}$ The protein levels of MGMT in U251-TR cells overexpressing miR-486-3p or miR-NC. d The mRNA levels of MGMT in U251-TR cells transfected with miR-486-3p or miR-NC. Each data point represents the mean \pm SEM from three independent experiments. $* P<0.05, * * P<0.01$

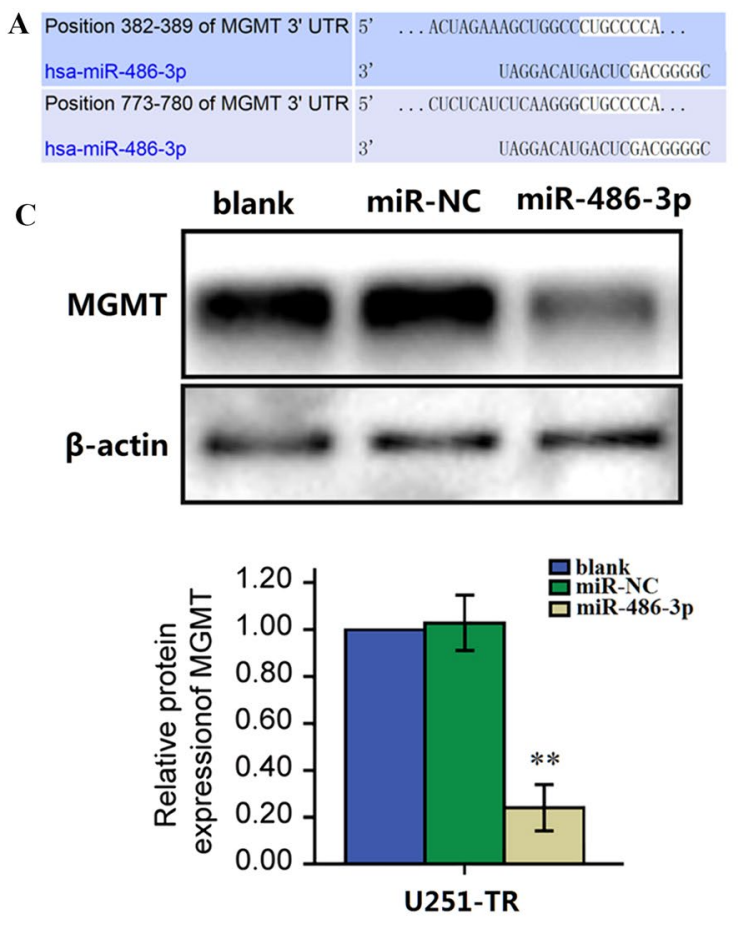

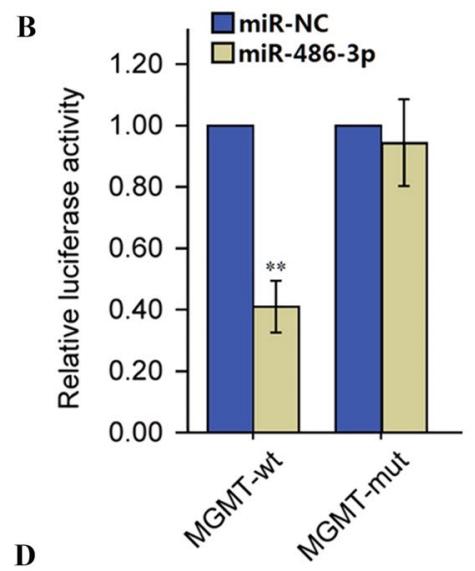

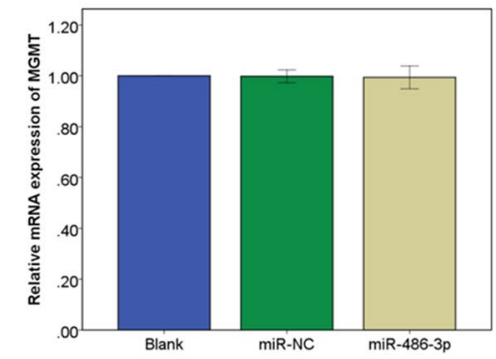

\section{MiR-486-3p Overexpression Enhances Temozolomide-Induced Growth Inhibition In Vivo}

In order to further confirm that MiR-486-3p overexpression enhances TMZ-induced growth inhibition, U251-TR cells were stably transfected with miR-486-3p, miR-486-3p-inhibitor, and miR-NC, respectively. CCK8 assay showed that after treatment with $30 \mu \mathrm{g} / \mathrm{ml}$ Allicin $+50 \mu \mathrm{g} / \mathrm{ml} \mathrm{TMZ}$ for 24 or $48 \mathrm{~h}$, the viability of U251-TR cells transfected with miR486-3p or miR-486-3p-inhibitor was significantly decreased compared with miR-486-3p-inhibitor group (Fig. 5a). Western blot showed that compared with miR-486-3p-inhibitor group, the expression of MGMT was decreased, whereas cleaved caspase- 3 (cleaved cas-3) and $\gamma$-H2AX expression were increased (Fig. 5b). In addition, after treatment with $30 \mu \mathrm{g} / \mathrm{ml}$ Allicin $+50 \mu \mathrm{g} / \mathrm{ml}$ TMZ for 24 or $48 \mathrm{~h}$, the activities of caspase-3 significantly increased (Fig. 5c). We also used tumor-bearing mice to investigate whether Allicin can reverse TMZ resistance in vivo. The survival curves showed that in miR-486-3p-overexpression group and miR-NC groups, the survival time was significantly increased compared with miR-486-3p-inhibitor group (Fig. 5d). Together, these data suggest that Allicin could upregulate miR-486-3p and then lead U251-TR cells sensitize to TMZ in vivo.

\section{Discussion}

Oral alkylating agent TMZ is a chemotherapeutic drug with remarkable effects on various solid tumors. At present, TMZ is listed as the first-line chemotherapeutic drug for glioblastoma. TMZ simultaneous radiotherapy combined with adjuvant chemotherapy is the standard treatment for glioblastoma. However, after years of clinical observation, its effect is still limited. The reasons may be as follows: First, the presence of the blood-brain barrier affects the distribution and absorption of the drug in the brain. Second, it may be due to tumor resistance genes, which made tumor cells lose sensitive to TMZ and drug resistance, resulting in tumor progression or recurrence (Arora and Somasundaram 2019; Shea et al. 2016). Therefore, it is meaningful to research the mechanism of TMZ resistance in glioblastoma and try to reverse it.

Previous study reported that Allicin suppresses the growth of certain cancers in vitro and in vivo (Dvořáková et al. 2015). Consistent with this report, our study showed that Allicin can increase the sensitivity of U251-TR to TMZ and promote glioblastoma cells apoptosis. Further study showed that the expression of miR-486-3p was significantly increased in Allicin treated group than control 
A

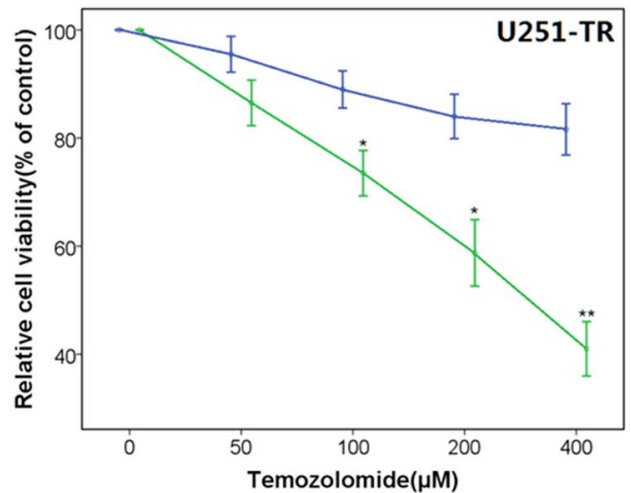

B

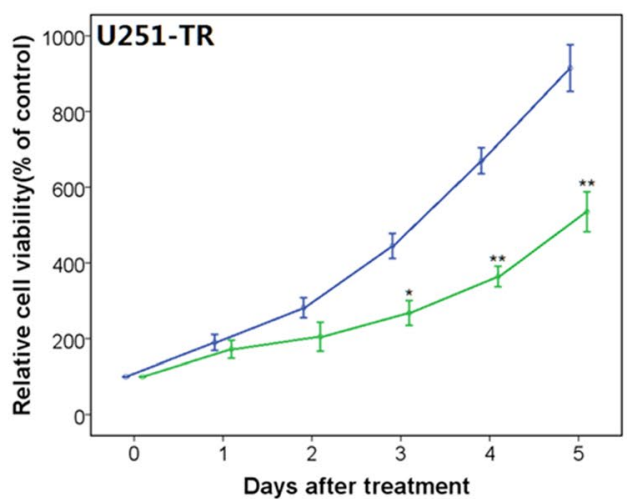

C

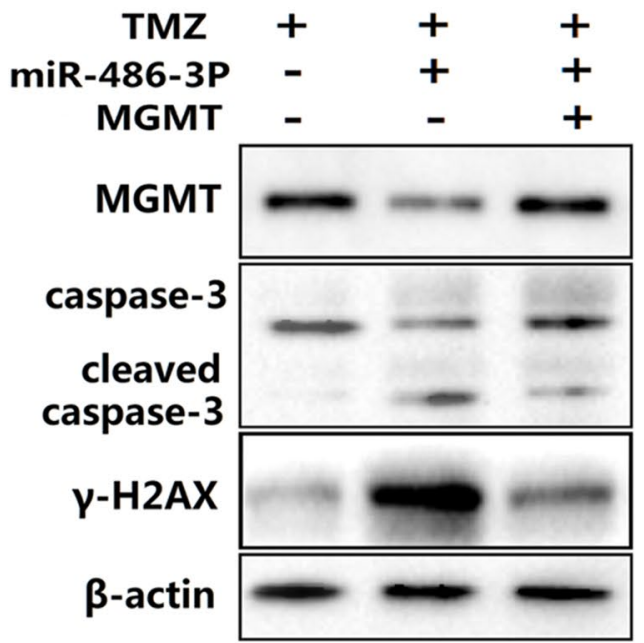

Fig. 4 miR-486-3p overexpression increases chemosensitivity to temozolomide. a Cell proliferation was evaluated in U251-TR and A172 cells stably expressing miR-NC or miR-486-3p with the TMZ treatments at different concentration. CCK8 assay was performed $48 \mathrm{~h}$ after treatment. b Cell proliferation in $200 \mu \mathrm{M}$ TMZ treatments was tested every $24 \mathrm{~h}$ in U251-TR and A172 cells overexpressing

group. Based on bioinformatics analysis, it is suggested that high expression of miR-486-3p may enhance the sensitivity of chemotherapeutic drugs in glioblastoma (Plaisier et al. 2016). The results of our study justify this hypothesis and found for the first time that Allicin can
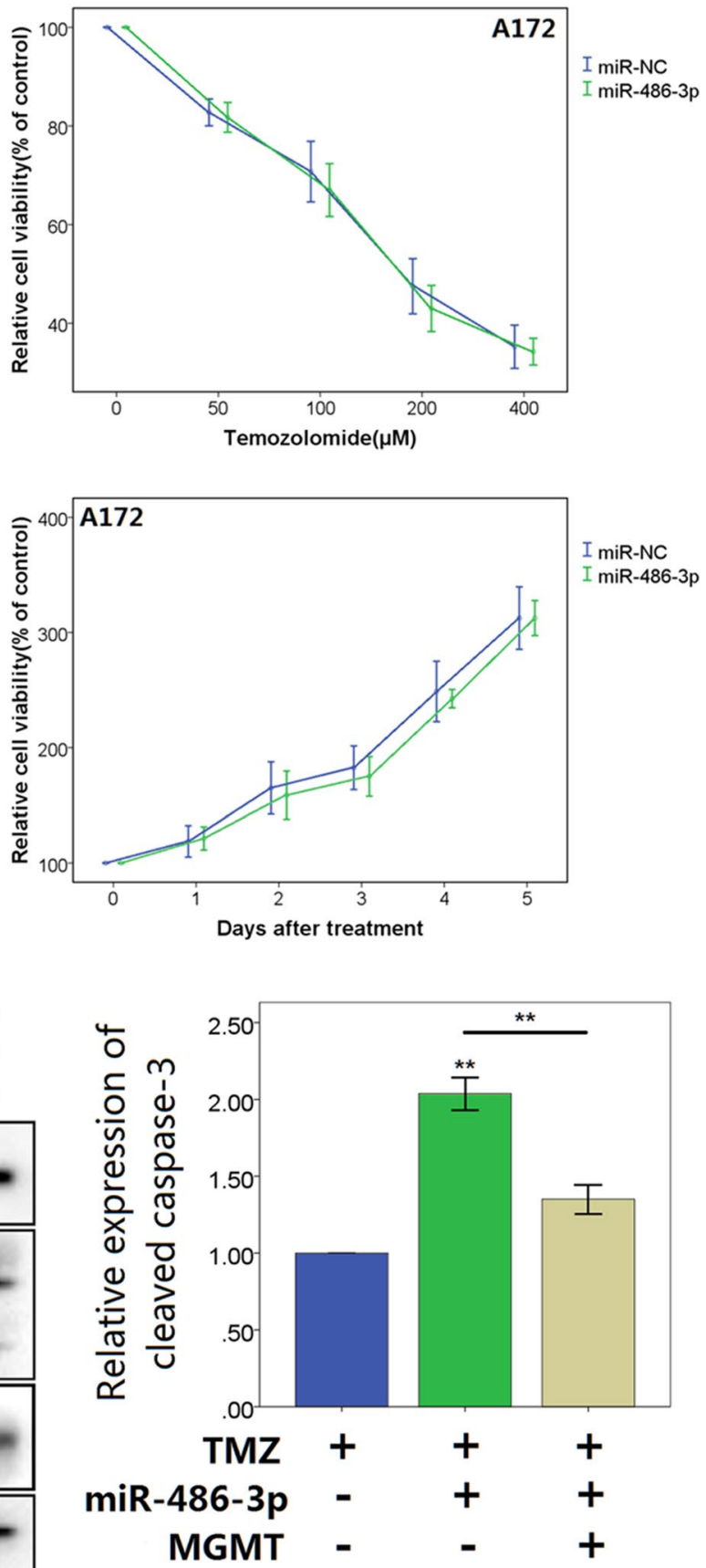

miR-486-3p and miR-NC. $\mathbf{c}$ Western blot analysis of MGMT, cleaved caspase-3, and $\gamma-\mathrm{H} 2 \mathrm{AX}$ expression in U251-TR cells co-transfected with miR-486-3p or miR-NC, and cultured in $200 \mu \mathrm{M}$ TMZ. The data represent results from one of three independent experiments. $* P<0.05, * * P<0.01$

promote glioblastoma cells apoptosis by promoting the high expression of miR-486-3p, reversing TMZ resistance. This finding complements previous research (Chu et al. 2012; Lawson and Wang 2005; Pan et al. 2018; Oommen et al. 2004) and partially clarifies the anti-tumor effect of 
A

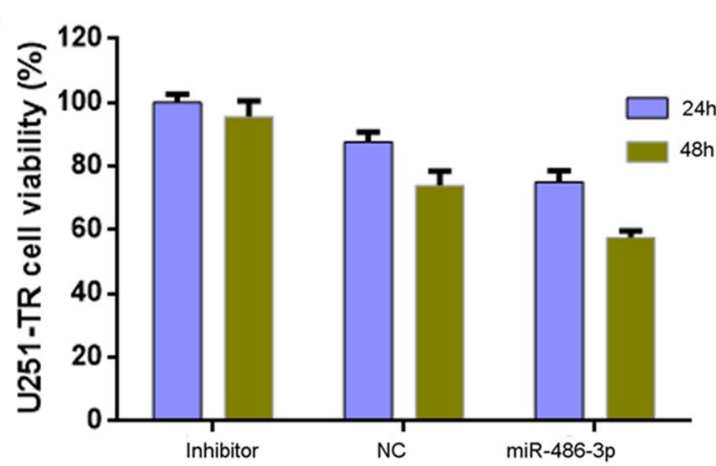

C

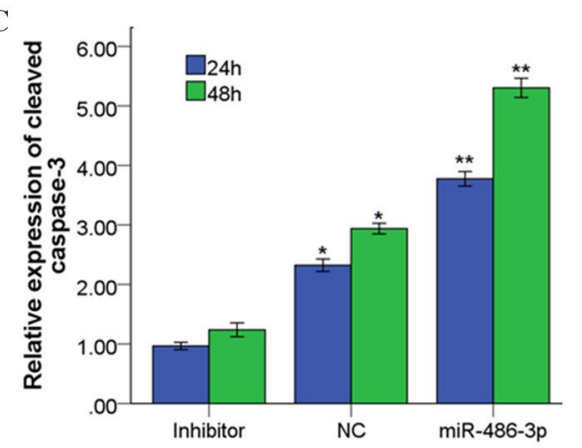

Fig. 5 Allicin promotes high expression of miR-486-3p that enhances TMZ sensitivity in tumor-bearing mice. a Overexpression miR486-3p reduced the U251-RT cells viability. Data presented as mean \pm SEM of cell viability from three independent experiments with triplicates in each experiment. b Western blot analysis confirmed that after $48 \mathrm{~h}$ treatment, miR-486-3p-overexpression group and miR$\mathrm{NC}$ group had a significant decrease in MGMT expression.c Expres-

Allicin. Nevertheless, its role in glioblastoma is poorly understood.

Many studies have demonstrated that the most popular mechanism of TMZ resistance is the expression of O6-methylguanine-DNA methyltransferase (MGMT) (Wick et al. 2014). MGMT protein is a kind of DNA repair enzyme, which can protect normal tissues from alkylating agent damage, reduce cancer, and also cause tumor tissue resistance to alkylating agent chemotherapy. There have been many studies on the effects of MGMT on TMZ resistance in glioblastoma treatment. Some researchers have found that cytotoxic drug withaferin A, which is involved in oxidative stress, can increase the sensitivity of glioblastoma to TMZ treatment by reducing MGMT expression and activating apoptosis induced by Akt/mTOR signaling pathway (Grogan et al. 2014). miR-200a-3p has also been found to interact with MGMT to increase susceptibility of glioblastoma to chemotherapy drugs (Berthois et al. 2014). Some studies found that liver-type glutaminase (LGA), which is transfected and expressed in human glioblastoma cell lines, causes a decrease in MGMT expression, and also increases sensitivity
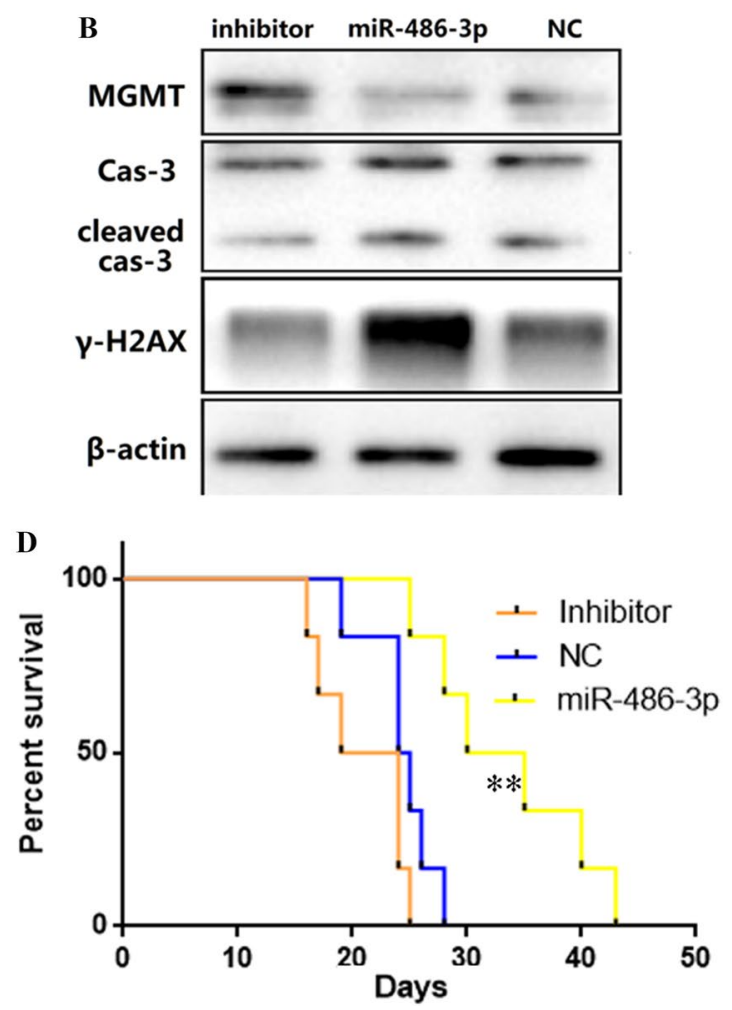

sion analysis of cleaved caspase- 3 showed that miR-486-3p overexpression group and miR-NC group promote apoptosis significantly. The data represent results from one of three independent experiments. d Survival curve of miR-486-3p overexpression, miR-486-3p inhibitor, and miR-NC control groups. $* P<0.05$, $* * P<0.01$ versus miR486-3p inhibitor group

of glioblastoma to chemotherapy drugs (Szeliga et al. 2012). And the expression of MGMT is upregulated by a variety of mechanisms, such as the acetylation of histones $\mathrm{H} 3$ and $\mathrm{H} 4$ (Nakagawachi et al. 2003), different nuclear transcription factors (AP-1, HIF-1 $\alpha, \mathrm{CEBP}, \mathrm{Sp} 1$, and NF- $\mathrm{B}$ ) (Bocangel et al. 2009), and the stabilization by binding of N-myc downstream regulated gene 1 protein (Weiler et al. 2014). The present study showed that miR-486-3p could directly bound to the 3'-UTR of MGMT, decreased the protein expression of MGMT through inhibiting the translation of the MGMT mRNA into the MGMT protein. Meanwhile, miR-486-3p overexpression rendered glioblastoma cells more sensitive to TMZ through targeting MGMT.

In summary, this study suggest that Allicin can increase the expression of miR-486-3p in glioblastoma, and miR486-3p inhibits protein translation of MGMT, which reverses TMZ resistance and promotes glioblastoma apoptosis in TMZ environment. Our study further elucidate the biological function of Allicin in glioblastoma. This biological function indicates that, in the future, Allicin can be used as adjunctive drug for TMZ to improve patient prognosis, 
and miR-486-3p may be a potential target for glioblastoma treatment, improving the effectiveness of comprehensive treatment.

Author Contributions CW designed this study. GGW and XL participated in animal study and data analysis. THZ, GGW and GJZ participated in collection of data. GGW, JGC, LC, MH and BLH revised the manuscript: $\mathrm{CW}$ provided financial support, $\mathrm{CZ}$ and $\mathrm{GGW}$ wrote the manuscript.

Funding This study was supported by Traditional Chinese Medicine Science Research Fund of Zhejiang Province (Grant No: 2020ZB108) and Zhejiang Province Department of Education Research Project Funding (Grant No: Y201942354).

\section{Compliance with ethical standards}

Conflicts of interest The authors declare no potential conflicts of interest in the study.

Ethical Approval All procedures performed in studies involving human participants and animals were in accordance with the ethical standards of the institutional and/or national research committee and with the 1964 Helsinki declaration and its later amendments or comparable ethical standards.

Open Access This article is licensed under a Creative Commons Attribution 4.0 International License, which permits use, sharing, adaptation, distribution and reproduction in any medium or format, as long as you give appropriate credit to the original author(s) and the source, provide a link to the Creative Commons licence, and indicate if changes were made. The images or other third party material in this article are included in the article's Creative Commons licence, unless indicated otherwise in a credit line to the material. If material is not included in the article's Creative Commons licence and your intended use is not permitted by statutory regulation or exceeds the permitted use, you will need to obtain permission directly from the copyright holder. To view a copy of this licence, visit http://creativecommons.org/licenses/by/4.0/.

\section{References}

Arora, A., \& Somasundaram, K. (2019). Glioblastoma vs temozolomide: Can the red queen race be won? Cancer Biology \& Therapy, 20(8), 1083-1090. https://doi.org/10.1080/15384047.2019.15996 62.

Berthois, Y., Delfino, C., Metellus, P., Fina, F., Nanni-Metellus, I., Al Aswy, H., et al. (2014). Differential expression of miR200a-3p and miR2 1 in grade II-III and grade IV gliomas: Evidence that miR200a-3p is regulated by $\mathrm{O}(6)$-methylguanine methyltransferase and promotes temozolomide responsiveness. Cancer Biology \& Therapy, 15(7), 938-950. https://doi.org/10.4161/cbt.28920

Bocangel, D., Sengupta, S., Mitra, S., \& Bhakat, K. K. (2009). p53-Mediated down-regulation of the human DNA repair gene O6-methylguanine-DNA methyltransferase (MGMT) via interaction with Sp1 transcription factor. Anticancer Research, 29(10), 3741-3750.

Borlinghaus, J., Albrecht, F., Gruhlke, M. C., Nwachukwu, I. D., \& Slusarenko, A. J. (2014). Allicin: Chemistry and biological properties. Molecules, 19(8), 12591-12618. https:// doi.org/10.3390/molecules190812591.

Bush, N. A., Chang, S. M., \& Berger, M. S. (2017). Current and future strategies for treatment of glioma. Neurosurgical Review, 40(1), 1-14. https://doi.org/10.1007/s10143-016-0709-8.

Chou, S. T., Peng, H. Y., Mo, K. C., Hsu, Y. M., Wu, G. H., Hsiao, J. R., et al. (2019). MicroRNA-486-3p functions as a tumor suppressor in oral cancer by targeting DDR1. Journal of Experimental \& Clinical Cancer Research, 38(1), 281. https://doi.org/10.1186/ s13046-019-1283-z.

Chu, Y. L., Ho, C. T., Chung, J. G., Rajasekaran, R., \& Sheen, L. Y. (2012). Allicin induces p53-mediated autophagy in Hep G2 human liver cancer cells. Journal of Agriculture and Food Chemistry, 60(34), 8363-8371. https://doi.org/10.1021/jf301298y.

Dvořáková, M., Weingartová, I., Nevoral, J., Němeček, D., \& Krejčová, T. (2015). Garlic sulfur compounds suppress cancerogenesis and oxidative stress: A review. Scientia Agriculturae Bohemica, 46(2), 65-72.

Grogan, P. T., Sarkaria, J. N., Timmermann, B. N., \& Cohen, M. S. (2014). Oxidative cytotoxic agent withaferin A resensitizes temozolomide-resistant glioblastomas via MGMT depletion and induces apoptosis through Akt/mTOR pathway inhibitory modulation. Investigational New Drugs, 32(4), 604-617. https ://doi.org/10.1007/s10637-014-0084-7.

Iorio, M. V., \& Croce, C. M. (2009). MicroRNAs in cancer: Small molecules with a huge impact. Journal of Clinical Oncology, 27(34), 5848-5856. https://doi.org/10.1200/JCO.2009.24.0317.

Jiang, M., Li, X., Quan, X., Yang, X., Zheng, C., Hao, X., et al. (2018). MiR-486 as an effective biomarker in cancer diagnosis and prognosis: A systematic review and meta-analysis. Oncotarget, 9(17), 13948-13958. https://doi.org/10.18632/oncotarget .24189 .

Kang, H. C., Kim, I. J., Park, J. H., Shin, Y., Ku, J. L., Jung, M. S., et al. (2004). Identification of genes with differential expression in acquired drug-resistant gastric cancer cells using high-density oligonucleotide microarrays. Clinical Cancer Research, $10(1 \mathrm{Pt}$ 1), 272-284.

Lawson, L. D., \& Wang, Z. J. (2005). Allicin and allicin-derived garlic compounds increase breath acetone through allyl methyl sulfide: Use in measuring allicin bioavailability. Journal of Agriculture and Food Chemistry, 53(6), 1974-1983. https://doi.org/10.1021/ jf048323s.

Nakagawachi, T., Soejima, H., Urano, T., Zhao, W., Higashimoto, K., Satoh, Y., et al. (2003). Silencing effect of CpG island hypermethylation and histone modifications on O6-methylguanineDNA methyltransferase (MGMT) gene expression in human cancer. Oncogene, 22(55), 8835-8844. https://doi.org/10.1038/ sj.onc.1207183.

Omura, N., Li, C. P., Li, A., Hong, S. M., Walter, K., Jimeno, A., et al. (2008). Genome-wide profiling of methylated promoters in pancreatic adenocarcinoma. Cancer Biology \& Therapy, 7(7), 1146-1156. https://doi.org/10.4161/cbt.7.7.6208.

Oommen, S., Anto, R. J., Srinivas, G., \& Karunagaran, D. (2004). Allicin (from garlic) induces caspase-mediated apoptosis in cancer cells. European Journal of Pharmacology, 485(1-3), 97-103. https://doi.org/10.1016/j.ejphar.2003.11.059.

Ostrom, Q. T., Gittleman, H., Xu, J., Kromer, C., Wolinsky, Y., Kruchko, C., et al. (2016). CBTRUS statistical report: Primary brain and other central nervous system tumors diagnosed in the United States in 2009-2013. Neuro-Oncology, 18(suppl_5), v1v75. https://doi.org/10.1093/neuonc/now207.

Pan, Y., Ma, P., Liu, Y., Li, W., \& Shu, Y. (2018). Multiple functions of m(6)A RNA methylation in cancer. Journal of Hematology \& Oncology, 11(1), 48. https://doi.org/10.1186/s13045-018-0590-8.

Plaisier, C. L., O'Brien, S., Bernard, B., Reynolds, S., Simon, Z., Toledo, C. M., et al. (2016). Causal mechanistic regulatory 
network for glioblastoma deciphered using systems genetics network analysis. Cell Systems, 3(2), 172-186. https://doi. org/10.1016/j.cels.2016.06.006.

Rahman, M., Reyner, K., Deleyrolle, L., Millette, S., Azari, H., Day, B. W., et al. (2015). Neurosphere and adherent culture conditions are equivalent for malignant glioma stem cell lines. Anatomy and Cell Biology, 48(1), 25-35. https://doi.org/10.5115/acb.2015.48.1.25.

Shankar, A., Kumar, S., Iskander, A. S., Varma, N. R., Janic, B., deCarvalho, A., et al. (2014). Subcurative radiation significantly increases cell proliferation, invasion, and migration of primary glioblastoma multiforme in vivo. Chinese Journal of Cancer, 33(3), 148-158. https://doi.org/10.5732/cjc.013.10095.

Shea, A., Harish, V., Afzal, Z., Chijioke, J., Kedir, H., Dusmatova, S., et al. (2016). MicroRNAs in glioblastoma multiforme pathogenesis and therapeutics. Cancer Medicine, 5(8), 1917-1946. https ://doi.org/10.1002/cam4.775.

Szeliga, M., Zgrzywa, A., Obara-Michlewska, M., \& Albrecht, J. (2012). Transfection of a human glioblastoma cell line with livertype glutaminase (LGA) down-regulates the expression of DNArepair gene MGMT and sensitizes the cells to alkylating agents. Journal of Neurochemistry, 123(3), 428-436. https://doi.org/10. 1111/j.1471-4159.2012.07917.x.
Weiler, M., Blaes, J., Pusch, S., Sahm, F., Czabanka, M., Luger, S., et al. (2014). mTOR target NDRG1 confers MGMT-dependent resistance to alkylating chemotherapy. Proceedings of the National Academy of Sciences of the United States of America, 111(1), 409-414. https://doi.org/10.1073/pnas.1314469111.

Wick, W., Weller, M., van den Bent, M., Sanson, M., Weiler, M., von Deimling, A., et al. (2014). MGMT testing-the challenges for biomarker-based glioma treatment. Nature Reviews Neurology, 10(7), 372-385. https://doi.org/10.1038/nrneurol.2014.100.

Xu, K., Zhang, Z., Pei, H., Wang, H., Li, L., \& Xia, Q. (2017). FoxO3a induces temozolomide resistance in glioblastoma cells via the regulation of beta-catenin nuclear accumulation. Oncology Reports, 37(4), 2391-2397. https://doi.org/10.3892/or.2017.5459.

Yang, S., Sui, J., Liu, T., Wu, W., Xu, S., Yin, L., et al. (2019). Expression of miR-486-5p and its significance in lung squamous cell carcinoma. Journal of Cellular Biochemistry, 120(8), 13912-13923. https://doi.org/10.1002/jcb.28665.

Publisher's Note Springer Nature remains neutral with regard to jurisdictional claims in published maps and institutional affiliations. 